\title{
Index
}

Note: Page numbers in italics refer to figures

(๑) indicates video clip in the BSAVA Library

120-degree rigid endoscopes 297-9

3D endoscopy 301-2

Abdominal insufflation see Insufflation

Acetabular fracture 259

Acute bradycardia after upper gastrointestinal (Gl) endoscopy 78

Adenocarcinoma

colonic $87,93-4,273$

gastric 74,75

intestinal 76,77

nasal $144-6$

Adrenalectomy 211-13

patient positioning 212

port placement 212

surgical technique 212-13

Aiming device for arthroscopy 241

Allergic rhinitis 143

Anaesthesia

and abdominal insufflation 115

for arthroscopy 242

for colonoscopy 89

and endosurgery 112-15, 285

for laparoscopy 113-15, 185

for oesophagoscopy 48

for respiratory tract endoscopy $99-100,112-13$

laser endosurgery 285

for rhinoscopy 113,134

for thoracoscopy $113,220-2,223,225,226,227,230$, 231,232

for upper gastrointestinal (GI) endoscopy 62

(see also Premedication/sedation)

Analgesia for arthroscopy 242

Antifoaming agents 62

Antral polyps $73,74,75$

Antrum 64-5

Arthroscopes 19, 237-8

for the carpus 256

for the elbow 250

for the hip 257

sheaths 20

for the shoulder 243

for the stifle 259-60

for the tarsus 263
Arthroscopy

anaesthesia 242

analgesia 242

antibiotics 242

arthroscopes 237-8

carpus

anatomy 256

instrumentation 256

joint examination 256

pathological conditions 257

patient preparation and positioning 256

procedure 256

elbow

anatomy 251

fragmented coronoid process (FCP) 251-3

humeral intracondylar fissure (HIF) 255-6

indications 250

instrumentation 250

osteoarthritis 255

osteochondritis dissecans (OCD) 253-4

patient preparation and positioning 250

procedure 250-1

ununited anconeal process (UAP) 254-5

fractures 257, 259, 265

hip

anatomy 258

biopsy 259

dysplasia 258-9

fractures 259

indications 257

instrumentation 257

pain 259

patient preparation and positioning 257

procedure 257-8

indications 236

instrumentation 236-41

accessory 238-40

aiming device 241

arthroscopes 237-8

cannulae 238-9

fluid management systems $240-1$

hand 239-40

optical system 237

power shavers 240

patient preparation and positioning 241-2

postoperative care 242

septic arthritis 265

shoulder

anatomy 242-3 
biceps tendon rupture 249

bicipital tenosynovitis (BTS) 248-9

glenohumeral ligament tears 247-8

incomplete ossification/osteochondral

fragmentation, caudal glenoid 247

indications 243

instrumentation 243

joint examination 244-5

medial instability 247

osteochondritis dissecans (OCD) 246-7

patient preparation and positioning 243

procedure 243-6

suspended limb 245-6

stifle

anatomy 261

cranial cruciate ligament injury 261-2 surgery 263

indications 259

instrumentation 259-60

medial meniscal transection 262

osteochondritis dissecans 262-3

patient preparation and positioning 260

procedure 260-1

tarsus

anatomy 264

indications 263

instrumentation 263

osteochondritis dissecans (OCD) 264

patient preparation and positioning 263

procedure 263-4

synovial biopsy 265

Aspergillosis $130,131,142,148-51$

laser endosurgery 290-1

Aspiration/lavage catheter 98

Auricular mass resection 225-6

Autoclavable endoscopes 133

Autofluorescence and enhanced contact endoscopy 301

Bacteraemia after upper gastrointestinal (GI) endoscopy 78

Bacterial rhinitis/sinusitis 143

Balloon dilation, oesophageal strictures $54-5$

Benign mucosal antral polyps 73, 74, 75

Benign nasal polyps 142

Benign nasal tumours 147

Biceps tendon rupture 249

Bicipital tenosynovitis (BTS) 248-9

Biofilm-related infections 164-5

Biopsy

in arthroscopy 265

in bronchoscopy 106

brush cytology 42

in colonoscopy $90,91-2$

in duodenoscopy 57,60,69, 70

in gastroscopy $57,66-7,74$

fine-needle aspiration 43,45

fluid collection 42

forceps $16,20,21,169$

selection $43-4,91$

intestinal 56-7, 193-4

in jejunoscopy 60,69

in laparoscopy 190-4

cholecystocentesis 191-2

intestine 56-7, 193-4

kidney 192-3

liver 190-1

pancreas 192 needles 99

in oesophagoscopy 49

in otoendoscopy 159

in rhinoscopy $136,140,146$

sample collection 43

sample processing 44-5

in thoracoscopy 233

in urethrocystoscopy/vaginoscopy 177

Bipolar electrosurgery 121

Bladder see Urinary bladder

Bougie dilators 55

Brachycephalic obstructive airway syndrome (BOAS) 101, 108, 109, 299

laser endosurgery 285-7

Breeding time assessment in the bitch $178-80$

Bronchi

examination 103

laser endosurgery 288-9

Bronchial collapse 271

Bronchoalveolar lavage (BAL) 103-5

Bronchoscopes 39

Bronchoscopy see Respiratory tract endoscopy; Tracheobronchoscopy

Brush cytology bronchial 105, 106 equipment 16,98 flexible endoscopy 42 gastric 67

Cameras $24-5,116,184$

capsular $9,70-1$

dual systems 300

high definition 301

Cannulae 19, 20-1

for arthroscopy 238-9

for laparoscopy 184-5, 188-9

placement 117-19

for thoracoscopy $219,222-3$

Capsule endoscopy $9,70-1$

Carbon dioxide insufflators 23, 32

Carbon dioxide lasers 282

Carcinoma

gastric 74,75

oesophageal 53

Cardia 66

Carpal fractures 257

Carpal synovitis 257

Carts 27, 30

Cervical catheterization 180-1

Cholecystectomy 207-8 transgastric 309

Cholecystocentesis 191-2

Cholesteatoma 154

Chondrosarcoma, nasal 146-7

Chromoendoscopy 301

Chronic inflammatory enteropathy $57,60,61,72,92-3$

Chylothorax 229-30

Client education 7

Coagulating electrode 16

Coccidioidomycosis 109

Colitis 87-8, 92-3

Colonic neoplasia 93-4

Colonic obstruction 273

Colonoscopes 35-9, 86-7

Colonoscopy see Lower gastrointestinal (GI) tract

Computed tomography (CT) 132-3, 153, 154-5, 171, 227, 229, 305 


\section{Constipation 94}

Cranial cruciate ligament (CCL) injury 261-2 arthroscopic-assisted surgery 263

Cranial mediastinal mass resection 226-7

Cryptorchidism 202-3

Cystitis 174

Cystoscopes 18, 19

Cystoscopy 203, 205, 293

-assisted guidewire placement 178 (see also Urethrocystoscopy/vaginoscopy)

Cystotomy, laparoscopic-assisted 203-5

Cytology brush 16, 98

Da Vinci robotic system 10, 303

Deep ulcers 73, 74, 76

Desufflation 126

Diaphragmatic hernia 209-10

Digital imaging 25-6

Diode lasers 283

Disinfection of instruments 29

Dislodger 16

Drains, thoracic 223

Duodenal biopsy 57, 60, 69, 70

Duodenal foreign bodies, removal 80

Duodenal neoplasia $75,76,77$

Duodenal ulceration 76,77

Duodenoscopy 57-60

biopsy $57,60,69,70$

fluid collection 69

indications 59

instrumentation 60

normal findings 72

pathological conditions 75-8

procedure 67-9

Duodenostomy tube placement 195

Duodenum

normal findings 72

pathological conditions $75-8$

Dynamic-range viewing endoscopes 299

Ear canal

abnormal findings 157-9

normal appearance 156

(see also Otoendoscopy)

Ectopic ureters 275-6

ablation 177-8, 276, 293-4

breed predisposition 170

Electrosurgery

bipolar 121

equipment 26

hazards 121-2

monopolar 120-1

uses for minimally invasive procedures 121

Endobronchial blockers (EBBs) 221-2

Endometrial disease 172

Endoscopes

care/cleaning 28-9

disinfection 29

dynamic-range 299

flexible 11

for colonoscopy $86-7$

comparison with rigid 9

controls $36-9$

handling 34, 35-6, 39

instrumentation 15-17

for oesophagoscopy 47 for respiratory tract endoscopy 98-9

for rhinoscopy 134, 137

selection 14-15

structure 12-14

for upper gastrointestinal (GI) endoscopy 60-1 for urethrocystoscopy/vaginoscopy 168-9

video versus fibre 14

history 1-5

light sources 22, 23

miniature 297

rigid

120-degree 297-9

for arthroscopy 237-8

for colonoscopy 87

comparison with flexible 9

handling 116-17

instrumentation 19-22, 117

with integrated working channels 297

for laparoscopy 116, 183-4

for oesophagoscopy 47

for otoendoscopy 152-3

for rhinoscopy 134, 137

selection 18-19, 116

structure 17-18

for thoracoscopy 116, 218-19

for urethrocystoscopy/vaginoscopy 168-9

viewing angles 17

semi-rigid 18,19

sterilization 29

storage $27,29-30$

(see also specific scopes)

Endoscopic submucosal dissection (ESD) 311-12

Endoscopy, basic techniques see Flexible endoscopy;

Rigid endoscopy and endosurgery; see also specific procedures

ENDOTIP cannula 19, 21

Enemas 88

Enhanced contact endoscopy 301

Enilconazole 149-50

Eosinophilic colitis 93

Eosinophilic enteritis 76

Ergonomics 27-8

Evolving trends in endoscopy 297-315

EZ-blocker ${ }^{\mathrm{TM}}$ 221, 222

'False middle ear' 161

Feeding tubes see Duodenostomy, Gastrostomy, Jejunostomy

Fibrescopes 12 comparison with video-endoscopes 14

Fine-needle aspiration 43, 45

Flexible endoscopes see Endoscopes and specific scopes

Flexible endoscopy

basic principles

biopsy $43-5$

brush cytology 42

fine-needle aspiration 43,45

fluid collection 42

handling 35-9

friction brakes $38-9$

handpiece $35-6$

insufflation/washing 37

steering wheels $37-8$

suction 37

valves/buttons $36-7$

video-endoscope controls 39 
health and safety $32-4$

endoscope 33-4

endoscopist 34

patient 33

image quality 31

instrumentation 11-17, 32

preparation 35

procedures 45

record keeping 45

(see also Video imaging systems)

use in a patient

advancing around flexures 41-2

advancing through sphincters 41

clearing the view 41

endoscope insertion 39-40

endoscope position 39

longitudinal rotation 40

operator position 39

red-out 40, 41, 42

steering the endoscope 40,46

video extras

escape of air bubbles during a leakage test of an endoscope 34

single- and two-handed manipulation of steering wheels 38

steering by tip deflection and torqueing 38

future developments 9-10, 297-315

lower gastrointestinal (Gl) tract 86-96

oesophagoscopy 47-55

respiratory tract $97-111$

upper gastrointestinal (GI) tract $56-85$

versus rigid 9

Fluid management systems for arthroscopy 240-1

Fluorescence techniques and targeted tissue dyeing 303-5

Fluoroscopy 171, 267, 270, 272, 274

Forceps

biopsy 16, 20, 21, 169

grasping 16, 19, 21, 53, 169, 239, 257

Foreign bodies

duodenal 80

gastric $79-80$

nasal $143-4$

nasopharyngeal 141

oesophageal 53-4

otic 158

removal

intestinal 196

nasopharyngeal 136

respiratory tract 110

upper gastrointestinal (Gl) tract $78-80$

respiratory tract 110

Foreign body rhinitis 143-4

Fractures 265

acetabular 259

carpal 257

Fragmented coronoid process (FCP) 251-3

Friction brakes 38-9

Frontal sinus exploration $140-1$

Fundus 66

Fungal rhinitis 148-51

Future developments in endoscopy $9-10,297-315$

Gastric biopsy 57, 66-7, 74

Gastric dilatation, after gastroscopy 78

Gastric foreign bodies $79-80$

Gastric neoplasia 73, 74, 75

Gastric overdistension 78
Gastric parasites 73,75

Gastric ulceration 73, 74, 75

Gastritis 73, 75

Gastroduodenoscopy 56

Gastroenterostomy 310

Gastrointestinal (GI) endoscopy see Lower gastrointestinal

(Gl) tract endoscopy; Upper gastrointestinal (GI) tract endoscopy

Gastrointestinal perforation 78

Gastrointestinal (Gl) tract see Lower gastrointestinal (Gl) tract endoscopy; Upper gastrointestinal (Gl) tract endoscopy

Gastro-oesophageal intussusception 51

Gastropexy

laparoscopic 195-6

transgastric 310

Gastroscopes 12, 13, 35-9

Gastroscopy

biopsy $57,66-7,74$

indications 57,59

instrumentation 60

normal findings $71-2$

pathological conditions $72-5$

procedure 63-7

theatre set-up 27,28

Gastrostomy tube placement $80-4$

Granuloma

oesophageal 52

respiratory tract 108,109

Granulomatous colitis 93

Haemorrhage 120, 292

after biopsy 190, 191, 192, 193

in Gl endoscopy 42, 73, 78

in rhinoscopy $138,139,151$

Haemostasis in endosurgery $22,119-22$

Half hitch knot 123-4, 126

Hand instruments, arthroscopy 239-40

Hasson (paediatric) technique 114-15, 187-8

Health and safety

ear flushing 161

endosurgery 112

lasers 284-5

Hiatal hernia 51

repair $210-11$

video extra

the endoscope in a j-flexed position looking at the lower oesophageal sphincter (abnormally dilated)

in a patient presenting with chronic vomiting.

A case of sliding hiatal hernia 51

Hip dysplasia 258-9

Hip pain 259

Histiocytic colitis 93

Holmium:YAG lasers 283

HOPKINS ${ }^{\circledR}$ endoscopes 17, 297

Humeral intracondylar fissure (HIF) 255-6

Hypertrophic pylorogastropathy 73, 74, 75

Idiopathic renal haematuria 278

lleocaecocolic junction 86

Ileoscopy 90

Image enhanced endoscopy 301

Incomplete ossification

caudal glenoid 247

humeral condyle 255-6

Inguinal hernia 210 
Instrument channels 13

Instrumentation

for arthroscopy 236-41

carpus 256

elbow 250

hip 257

shoulder 243

stifle 259-60

tarsus 263

for colonoscopy 86-7

for flexible endoscopy 15-17

for foreign body removal $53,78-9$

for haemostasis 119-20, 219

for interventional endoscopy and radiology 267-9

for laparoscopy 183-5

for laser endosurgery

respiratory tract 285,288

for oesophagoscopy 47

for otoendoscopy 152-3

for respiratory tract endoscopy $98-9$

for rhinoscopy 133

anterior 137

caudal 134

for rigid endoscopy $19-22,117$

specialized

120-degree rigid endoscopes 297-9

dynamic-range viewing endoscopes 299

miniature endoscopes 297

rigid endoscopes with integrated working channels 297

for thoracoscopy 218-20

for upper gastrointestinal (Gl) tract endoscopy 60-1

for urethrocystoscopy/vaginoscopy 168-9

(see also Endoscopes and other specific instruments)

Insufflation

abdominal 114-15

anaesthetic considerations 115, 185

complications 114-15, 214

equipment $23-4,32,184$

in flexible endoscopy $13,37,41,62,66$

technique

comparison $187-8$

Hasson (paediatric) 114-15, 187

Veress needle 114, 186-7

thoracic 221

Integrated advanced imaging modalities, endoscopy and 305

Interventional endoscopy and radiology

equipment $267-9$

for gastrointestinal conditions

colonic obstructions 273

oesophageal strictures 272-3

for respiratory conditions

bronchial collapse 271

nasopharyngeal stenosis (NPS) 271-2

tracheal collapse 270-1

for urinary tract conditions

ectopic ureters 275-6

idiopathic renal haematuria 278

refractory urethral sphincter mechanism incompetence 278

ureteral obstruction $276-7$

urethral obstruction 278

vascular interventions

congenital portosystemic shunts (PSSs) 274-5

interventional oncology 273-4

intrahepatic shunt embolization 274-5

percutaneous transvenous embolization (PTE) 275
Intestinal adenocarcinoma 76, 77

Intestinal biopsy $56-7,193-4$

Intestinal parasites $75,77-8$

Intrahepatic shunt embolization 274-5

Intranasal airway obstruction 287

Intussusception

gastro-oesophageal 51

ileocolic/ileocaecal 94

Irrigation 13, 122, 152, 155

Irritable bowel syndrome (IBS) 93

Jejunoscopy

indications 60

procedure 69

Jejunostomy tube placement 85

Knot-tying

extracorporeal 123

intracorporeal 123-6

Laparoscopes 18, 19

Laparoscopy

adrenalectomy 211-13

anaesthesia 113-15, 185

biopsy

cholecystocentesis 191-2

intestine 193-4

kidney 192-3

liver 190-1

pancreas 192

cancer staging 213

cholecystectomy 207-8

closure 126-7

complications 213-15

conversion to open surgery 215

cryptorchid surgery/vasectomy 202-3

cystotomy 203-5

endoscope choice 116

feeding tube placement 195

foreign body removal, intestinal 196

gastropexy 195-6

hernia repair 209-11

history 3-5

indications 183

instrumentation $23,183-5$

insufflation

Hasson (paediatric) technique 114-15, 187

Veress needle technique $114,186-7$

laser endosurgery 291-5

miniature laparoscopy and needle endoscopy 299-300

ovarian remnant removal 201-2

ovariectomy 199-201

ovariohysterectomy 197-9

patient positioning 116

patient preparation 185

port placement 117-19, 188, 189

for adrenalectomy 212

for cholecystectomy 207

for cystotomy 203

for hernia repair 209, 210, 211

for intestinal biopsy 194

for liver biopsy 191

for ovariohysterectomy 197

for splenectomy 206

for ureteronephrectomy 208 
postoperative care 127

procedure 186-90

splenectomy 206-7

suction/irrigation 122

theatre set-up 28

transanal (transcolonic) 310

transgastric 308-10

transvaginal 306-8

transvesical 310

ureteronephrectomy 208-9

video extra

passing instruments around the caudal edge of the falciform fat 201

Laryngeal collapse 286

Laryngeal mass 287-8

Laryngeal paralysis 101, 102

Larynx

examination 101-2

laser endosurgery 287-8

Laser-assisted turbinectomy (LATE) 287, 288

Lasers

endosurgery

respiratory tract 285-91

urinary tract $291-5$

health and safety $284-5$

physics 280-2

and tissue interactions 283-4

types

carbon dioxide 282

diode 283

holmium:YAG 283

neodymium:YAG 282-3

thulium:YAG 283

in urethrocystoscopy $177-8$

Lateral glenohumeral ligament tears $247-8$

Leakage testing 13

Leiomyoma, oesophageal 52

Ligamentum arteriosum division 230-2

LigaSure $^{\mathrm{TM}}$ vessel sealer 21,26

Light sources 22, 23, 184

Lithotripsy $177,178,291-3$

Liver biopsy 190-1

Lower gastrointestinal (Gl) tract endoscopy anaesthesia 89

anatomy 86

biopsy $90,91-2$

complications 96

indications $87-8$

instrumentation

flexible endoscopes $86-7$

rigid endoscopes 87

pathological conditions

caecal disorders 87, 94

colitis 87

eosinophilic 93

granulomatous 93

histiocytic 93

lymphocytic-plasmacytic 92-3

colonic obstruction 273

constipation 94

intussusception 87, 94

irritable bowel syndrome 93

neoplasia $87,93-4$

rectal tumours 95

rectal diverticulum 87,95

rectal polyps 87,95

rectal strictures 87,95

patient positioning 89 patient preparation 88

postoperative care 96

premedication 89

procedure 89-91

Lung lobectormy 227-9

Lymph nodes, excision 233

Lymphangiectasia 75, 77

Lymphocytic-plasmacytic colitis 92-3

Lymphoid follicles, reproductive tract 171

Lymphoid hyperplasia 136, 141

Lymphoma 298

colonic $93-4$

duodenal 76

gastric 75

nasal 144

Lymphoplasmacytic enteritis 76

Lymphosarcoma

duodenum 77

nasal 142,145

renal 193

Magnetic resonance imaging (MRI) 8, 10, 153, 155

Marketing endoscopy services 7

Masses

cranial mediastinal 226-7

laryngeal/pharyngeal $287-8$

nasal 289-90

otic 159

right auricular 225-6

tracheobronchial 288

(see also Neoplasia)

Medial meniscus injury 262

Medial shoulder instability 247

Mediastinal biopsy 233

Mediastinal mass resection 226-7

Megaoesophagus 52

Melanoma 145, 146

Middle ear, abnormal findings 161

Miniature endoscopes 297

Miniature laparoscopy 299-300

Monitors 14, 22, 25

Monopolar electrosurgery 120-1

Multi-Purpose Rigid ${ }^{\text {TM }}$ (MPR) endoscopes 18, 20

Myringotomy 163-4

complications 166

Nasal cavity and nasopharynx, laser endosurgery 289-91

Nasal masses 289-90

Nasopharyngeal polyps 142, 145, 289-90

Nasopharyngeal sialocoeles 286

Nasopharyngeal stenosis (NPS) 143, 271-2, 290, 291

Natural orifice transluminal endoscopic surgery (NOTES) 9-10, 305-11

approach $306,307,308$

hybrid procedures 300,311

transanal (transcolonic) laparoscopy 310

transgastric laparoscopy 308-10

cholecystectomy 309

gastroenterostomy 310

gastropexy 310

ovariectomy 309

transoesophageal approach to the thorax 310-11

transvaginal laparoscopy and endosurgery 306-8

ovariectomy/ovariohysterectomy

in cats 308

in dogs $306-8$

transvesical laparoscopy 310 
Needle endoscopy 299-300

Neodymium:YAG lasers 282-3

Neoplasia

colonic 93-4

duodenal $75,76,77$

gastric $73,74,75$

hepatic 304

intrathoracic 233

nasal 144-8

oesophageal $52-3$

otic 159

staging 145, 213, 233

urinary bladder/urethra 175

urinary tract 273

vaginal 172

(see also specific tumours)

NOTES see Natural orifice transluminal endoscopic surgery

Oesophageal achalasia 52

Oesophageal diverticula 53

Oesophageal foreign bodies 53-4

Oesophageal neoplasia 52-3

Oesophageal stricture $54-5,272-3$

Oesophagitis $50-1$

Oesophagoscopy

anaesthesia 48

complications 55

contraindications 48

indications $47-8$

instrumentation 47

normal findings $49-50$

pathological conditions

diverticula 53

foreign bodies 53-4

gastro-oesophageal intussusception 51

hiatal hernia 51

motility disorders 52

neoplasia $52-3$

oesophagitis $50-1$

strictures $54-5,272-3$

vascular ring anomalies 52

patient positioning 48

patient preparation 48

premedication 48

procedure 48-9

video extra

the endoscope in a j-flexed position looking at the lower oesophageal sphincter (abnormally dilated) in a patient presenting with chronic vomiting. A case of sliding hiatal hernia 51

Ollulanus tricuspis 75

One-lung ventilation (OLV) 221-2

Oslerus osleri 108, 109

Osteoarthritis

elbow 255

tarsus 265

Osteochondritis dissecans (OCD)

elbow 253-4

shoulder $246-7$

stifle 262-3

tarsus 264

Osteosarcoma, nasal 145, 147, 148

Otic discharge 157-8

Otic foreign bodies 158

Otic masses 159
Otitis externa 157-8, 160, 161, 162, 165

Otitis media 160, 161, 163, 164-6

Otoendoscopy

abnormal findings 157-61

anaesthesia 155-6

biofilm-related infections

diagnosis $164-5$

management 165

cleaning/flushing procedures

flushing 162-3

preliminary ear cleaning 162

sampling 161

complications 166

ear canal

foreign bodies 158

masses 159

otitis externa and discharge 157-8

indications 153

instrumentation 152-3

middle ear 161

myringotomy 163-4

normal findings $156-7$

patient positioning 155-6

patient preparation 153

postoperative care 165-6

premedication 155-6

preoperative diagnostic investigations $153-5$

procedure 156

tympanic membrane $160-1$

video extras

flushing technique: appearance of the intact tympanic membrane after the flushing procedure 163

flushing technique: flushing and suctioning cycles in the external ear canal of a dog with otitis externa 162

foreign bodies: removal of plant awns using grasping forceps passed through the working channel of the video-otoscope 158

myringotomy procedure: the incision into the caudoventral quadrant of the pars tensa is made with a 3.5 Fr tomcat catheter 164

removal of an inflammatory polyp from the ear canal of a cat 159

Otoscopes 18, 23, 152-3

Ovarian remnant removal 201-2

Ovariectomy

laparoscopic 199-201

transgastric 309

transvaginal 306-8

Ovariohysterectomy

laparoscopic 197-9

patient positioning 116

transvaginal $306-8$

Pancreatic biopsy 192

Paramesonephric septum 171-2, 174 laser endosurgery 295

Parasites

gastric 73,75

intestinal $75,77-8$

(see also individual species)

Patient assessment/stabilization 8

Percutaneous endoscopic gastrostomy (PEG) tube

placement

contraindications 80

indications 80 
instrumentation 81

method 81-4

principle 80,81

problems and failures 84

removal 84

Percutaneous transvenous embolization (PTE) 275

Pericardiectomy 223-5

Pericardiocentesis 224

Peristalsis 64,65

Peroral endoscopic myotomy (POEM) 312

Persistent hymen 171-2

Persistent right aortic arch division 230-2

Peyer's patches 72

Pharyngeal mass 287-8

Pharyngotomy 136-7

Pharynx

examination 100

laser endosurgery 287-8

Physaloptera 73, 75

Pleural biopsy 233

Pneumoperitoneum 113-14

Pneumothorax 113, 227

Polyps

antral $73,74,75$

nasopharyngeal $142,145,289-90$

otic 159

rectal 87,95

removal 85, 289-90, 295

urinary bladder/urethra 205, 295

vaginal 172

Port placement 117-19

arthroscopy

carpus 256

elbow 250-1

hip 257-8

shoulder 243-4

stifle 260-1

tarsus 263, 264

laparoscopy 188

adrenalectomy 212

cholecystectomy 207

cystotomy 203

hernia repair 209, 210, 211

ovariectomy 199-201

ovariohysterectomy 197

splenectomy 206

ureteronephrectomy 208

thoracoscopy 222-3

auricular mass resection 225

chylothorax management 230

complications 233-4

lung lobectomy 228

mediastinal mass resection 226

pericardiectomy 223

persistent right aortic arch division 231

pyothorax management 232

Portosystemic shunts (PSSs) 274-5

Postoperative care 127

after arthroscopy 242

after bronchoscopy 110

after colonoscopy 96

after otoendoscopy 165-6

after rhinoscopy 151

after urethrocystoscopy/vaginoscopy 181

Power shavers 26-7, 240

Premedication/sedation

for colonoscopy 89

for oesophagoscopy 48 for otoendoscopy 155-6

for respiratory tract endoscopy 99-100, 113

for rhinoscopy 134

for upper gastrointestinal (GI) endoscopy 61

Pressure valve 13

Probes 286

arthroscopic 239

palpation 21, 116, 120, 185, 191, 193

Proctoscopy 33

Proliferative urethritis 175

Prostatic disease 172

Pseudomonas aeruginosa 162, 165

Pumps 23-4

Pyloric intubation 68

Pyloric stenosis 73, 75

Pyothorax 232-3

Radiography 56, 107, 132, 153, 171, 226, 254, 270

Rectal diverticulum 87, 95

Rectal polyps 87, 95

Rectal strictures 87,95

Red-out 40

Refractory urethral sphincter mechanism incompetence 278

Remnant hymen $171-2$

Renal biopsy 192-3

Reporting proforma for endoscopy $58-9$

Respiratory tract endoscopy

anaesthesia 99-100, 112-13

complications $110-11$

contraindications 98

foreign body removal 110

indications $97-8$

instrumentation 98-9

interventional endoscopy and radiology 270-2

laser endosurgery 285-91

pathological conditions 106-9

brachycephalic obstructive airway syndrome 108

tracheal collapse 107-8

tracheobronchitis 106-7

patient positioning 100

postoperative care 110

premedication 99-100

procedures

biopsy 106

bronchoalveolar lavage 103-5

brush cytology 105-6

retropharyngeal posterior rhinoscopy 100

tracheobronchoscopy 100-3

video extras

bilateral laryngeal paralysis in a cat 102

endoscopic removal of a grass stalk foreign body from the airway of a dog 110

epiglottic retroversion - intermittent spontaneous retroflexion of the epiglottis during inspiration

causing obstruction of the rima glottides 101

grade III collapsing trachea in a Chihuahua 103

laryngeal carcinoma in a cat 102

Retropharyngeal posterior rhinoscopy 100

Rhinitis 141

allergic 143

bacterial 143

foreign body $143-4$

fungal $148-51$

ulcerative 139

Rhinoscope 18 
Rhinoscopy

anaesthesia 113, 134

anatomical considerations 128-9

anterior

biopsy 140

instrumentation 137

normal appearance 138,139

patient positioning 137

patient preparation 137

procedure 137-9

caudal

biopsy 136

foreign body removal 136

instrumentation 134

lateral pharyngotomy 136-7

nasal swabs 136

normal appearance 135-6

patient positioning 135

patient preparation 135

procedure 135

clinical examination 130-1

clinical history 130

complications 151

differential diagnoses 130

frontal sinus exploration 140-1

indications 129

instrumentation 133, 134, 137

intraoperative diagnostic investigations 132-3

pathological conditions

allergic rhinitis 143

bacterial rhinitis/sinusitis 143

foreign body rhinitis $143-4$

fungal rhinitis 148-51

nasopharynx

foreign body 141

fungal infection 142

lymphoid hyperplasia 141

polyps 142

stenosis 143

neoplasia

adenocarcinoma $144-6$

benign 147

chondrosarcoma 146-7

lymphoma 144

lymphosarcoma 145

melanoma 146

osteosarcoma 147, 148

patient positioning 116

postoperative care 151

premedication 134

preoperative diagnostic investigations 131

retropharyngeal posterior 100

Right auricular mass resection 225-6

Rigid endoscopes see Endoscopes and specific scopes

Rigid endoscopy and endosurgery

arthroscopy 236-66

basic principles

abdominal insufflation 114-15

anaesthesia

laparoscopy 113-15

respiratory tract $112-13$

thoracoscopy 113

choice of endoscope 116

closure 126-7

desufflation 126

dissection and haemostasis 119-22

electrosurgery 120-2

bipolar 121 hazards $121-2$

monopolar 120-1

endosurgery considerations 116-17

health and safety 112

instrumentation 19-22, 117

knot-tying techniques

extracorporeal 123, 124

intracorporeal 123-6

patient positioning 116

port placement 117-19

postoperative care 127

specimen retrieval $122-3$

suction and irrigation 122

future developments 9-10, 297-315

laparoscopy 183-217

otoendoscopy 152-67

rhinoscopy 128-51

thoracoscopy 218-35

urethrocystoscopy/vaginoscopy 168-82

versus flexible 9

$\mathrm{ROBI}{ }^{\circledR}$ plus, endoscope system 26

Robotic systems 10, 302-3

Rod-lens system 17

Roeder knot 123, 124

Scissors 16

Sedation see Premedication/sedation

Septic arthritis 252, 265

Shavers 26-7, 240

Sheaths 18, 19-20

Single-incision ports 21

Sinonasal aspergillosis (SNA) 150-1

Sino-orbital aspergillosis (SOA) 150-1

Snares 16

Soft palate elongation 286

Specimen retrieval bag 220

Spirocerca lupi 52

Splenectomy 206-7

Squamous cell carcinoma 52, 145

Stenotic ureteral openings, laser endosurgery 295

Sterilization 29

Stomach

normal findings $71-2$

pathological conditions $72-5$

Suction 13, 24, 32, 36, 37, 122, 153, 162-3

Superficial ulcers 73,74

Surgical glue 10

Surgical staplers 219-20

Synovial biopsy, tarsal 265

Taenia spp. 75

Teamwork 27-8

Telescopes 17, 218-19

Theatre set-up 27-8

Thoracic duct ligation 229-30, 300, 304

Thoracoscopy

access and port placement 222-3

anaesthesia 113, 220-2, 223, 225, 226, 227, 230, 231, 232

biopsy 233

complications 233-4

indications 218

instrumentation 218-20

and oncological staging 233

patient positioning 116

patient preparation 220 
surgical procedures

chylothorax management 229-30

cranial mediastinal mass resection 226-7

lung lobectomy 227-9

lymph node excision 233

pericardiectomy 223-5

persistent right aortic arch division 230-2

pyothorax management 232-3

right auricular mass resection 225-6

and thoracic drain placement 223

video extras

demonstration of the correct bronchoscopically guided placement of an endobronchial blocker 221

optical entry using a trocarless cannula (ENDOTIP, Karl Storz Endoscopy) 22

resection of a cranial mediastinal mass and removal from the thorax in a specimen retrieval bag 220

resection using an endoscopic stapler of a consolidated lung lobe in a dog secondary to pneumonia associated with chronic grass awn migration 228

thoracic duct ligation with haemoclips 230

Three-dimensional (3D) endoscopy 301-2

Thulium:YAG lasers 283

Tonsillar enlargement 287

Toxocara 75, 77

Trachea

examination $102-3$

laser endosurgery 288-9

Tracheal collapse $107-8,270-1$

Tracheal granulation 289

Tracheal hypoplasia 109

Tracheal stent 108

Tracheobronchial mass 288

Tracheobronchitis 106-7

Tracheobronchoscopy

anaesthesia 112-13

procedure 100-3

(see also Respiratory tract endoscopy)

Transanal (transcolonic) laparoscopy 310

approach 306

video extra

transanal laparoscopy in a dog 31

Transgastric laparoscopy 308-10

approach 306, 308

cholecystectomy 309

gastroenterostomy 310

gastropexy 310

ovariectomy 309

video extras

closure of the transgastric laparocentesis site using an over-the-top technique 309

dog 1 year after transgastric laparoscopy with closure of the laparocentesis site using endoscopic clips 309

transgastric approach to the abdominal cavity in a dog using a hybrid NOTES procedure 311

transgastric cholecystectomy in a dog 309 transgastric gastropexy in a dog (case 1) 310 transgastric gastropexy in a dog (case 2) 310 transgastric ovariectomy in a dog 309

Transmission of infection, upper gastrointestinal (GI) endoscopy 78

Transoesophageal approach to the thorax $306,310-11$ video extra

peroral thoracoscopy in a cat 311
Transvaginal laparoscopy and endosurgery 306-8

approach 306, 307

ovariectomy/ovariohysterectomy

in cats 308

in dogs $306-8$

video extras

transvaginal approach to the abdomen using a flexible endoscope 306

transvaginal ovariectomy in a cat using a diode laser 308

transvaginal ovariectomy in a dog 307

transvaginal ovariectomy in a dog using an optical trocar 307

transvaginal ovariohysterectomy in a dog 308

transvaginal ovariohysterectomy in a dog using a hybrid NOTES procedure 311

Transvesical laparoscopy 310 approach 306

Trichuris spp. 78

Trocars 18, 19, 20, 184-5

Turbinates and intranasal airway obstruction 287

Turbinectomy 287, 288

Tympanic membrane, abnormal findings 160-1 (see also Otoendoscopy)

Ulcerative rhinitis 139

Ulcers

duodenal 76,77

gastric $73,74,75$

Ultrasonography 8, 153, 171, 248

Uncinaria 75, 78

Ununited anconeal process (UAP) 254-5

Upper gastrointestinal (GI) tract endoscopy

antral polyps $73,74,75$

biopsy 57,70

capsule endoscopy $9,70-1$

complications

acute bradycardia 78

bacteraemia 78

decreased venous return from gastric overdistension 78

gastric dilatation 78

gastrointestinal perforation 78

mucosal haemorrhage 78

transmission of infection 78

contraindications 60

crypt abscessation 75,77

duodenum

normal findings 72

pathological conditions 75-8

gastritis 73,75

gastroduodenoscopy 56

hypertrophic pylorogastropathy 73, 74, 75

indications $56-60$

biopsy 57

duodenoscopy 57-60

full upper gastrointestinal tract endoscopy 57

gastroscopy 57,59

jejunoscopy 60

reporting proforma $58-9$

inflammatory enteropathy $75,76,77$

instrumentation 60-1

jejunostomy tube placement 85

limitations 56

lymphangiectasia 75,77

neoplasia 73, 74, 75, 76, 77 
normal findings duodenum 72 stomach $71-2$ parasites $73,75,77-8$

pathological conditions antral polyps $73,74,75$

crypt abscessation 75,77

duodenum 75-8

gastritis 73,75

hypertrophic pylorogastropathy $73,74,75$

inflammatory enteropathy $75,76,77$

lymphangiectasia 75,77

neoplasia $73,74,75,76,77$

parasites $73,75,77-8$

pyloric stenosis 73,75

stomach $72-5$

ulcers 77

benign $74,75,76$

deep $73,74,76$

superficial 73,74

patient management

anaesthesia 62

antifoaming agents 62

monitoring 62

patient positioning 62

premedication 61

recovery 62-3

patient preparation 61

percutaneous endoscopic gastrostomy (PEG) tube

placement

contraindications 80

indications 80

instrumentation 81

method 81-3

principles 80,81

problems and failures 84

removal 84

use $83-4$

polyp removal 85

procedure 63-70

duodenoscopy 67-9, 70

ending the examination 69

gastroscopy 63-7

jejunoscopy 69

mouth gag 63

pyloric stenosis 73,75

role in investigating gastrointestinal disease 56

stomach

normal findings 71-2

pathological conditions $72-5$

therapeutic procedures

complications 80

contraindications 80

foreign body removal $78-80$

duodenal 80

gastric $79-80$

instrumentation $78-9$

ulcers 77

benign $74,75,76$

deep $73,74,76$

superficial 73,74

video extras

placement of a percutaneous endoscopic gastrostomy (PEG) tube in a cat 82 upper $\mathrm{Gl}$ endoscopy 68

upper Gl endoscopy of a dog with a gastric carcinoma 75 video sequence recorded from an Alicam ${ }^{\circledR}$ video endoscopy capsule, showing a bleeding gastric polyp 71

Ureteral ectopia 173-4, 177-8

Ureteral obstruction $276-7$

Ureterocoeles 174 laser endosurgery 295

Ureteronephrectomy patient positioning and port placement 208 surgical technique 208-9

Ureters, pathological conditions 173-4

Urethra, pathological conditions 172-3, 174-5

Urethral dyssynergia 173

Urethral neoplasia 175

Urethral obstruction 173, 278

Urethral sphincter mechanism incompetence (USMI) 172-3

Urethrocystoscopy/vaginoscopy 302 breeding time assessment in the bitch 178-80 cervical catheterization 180-1 clinical examination 170 clinical history 169-70 complications 181 indications 169 instrumentation 168-9 intraoperative diagnostic imaging 171 operative techniques

biopsy 177

cystoscopy-assisted placement of a ureteral guidewire 178

ectopic ureter ablation 177-8

laser lithotripsy 177,178

patient positioning 116

postoperative care 181

preoperative diagnostic investigations 170-1

procedure

females $175-6$

males 177

prostatic disease 172

ureters

ectopia 173-4, 177-8

obstruction $276-7$

ureterocoeles 174

urethra

chronic infection 174

dyssynergia 173

neoplasia 175

obstruction 173, 278

proliferative urethritis 175

urethral sphincter mechanism incompetence (USMI) 172-3

urolithiasis $174-5$

urinary bladder

chronic infection 174

neoplasia 175

urolithiasis $174-5$

vagina

cervical abnormalities 172

endometrial disease 172

hyperplasia 172

neoplasia 172

polyps 172

vestibule

lymphoid follicles 171

paramesonephric septum 171-2

remnant/persistent hymen 171-2

vestibulovaginal stenosis 172 
video extras

normal urethrocystoscopy of the neutered bitch

176

normal urethrocystoscopy of the neutered male dog

$$
177 \bullet
$$

Urethroliths 175, 292

Urethroscopy see Urethrocystoscopy/vaginoscopy

Urinary bladder, pathological conditions 174-5

Urinary tract

infection (UTI) 174

interventional endoscopy and radiology

ectopic ureters 275-6

idiopathic renal haematuria 278

refractory urethral sphincter mechanism incompetence 278

ureteral obstruction $276-7$

urethral obstruction 278

laser surgery 291-5

neoplasia 273

Urolithiasis $169,171,174-5$

Uroliths 170, 175

laser lithotripsy 291-3

Vagina, pathological conditions 172

Vaginal hyperplasia 172

Vaginal neoplasia 172

Vaginal polyps 172

Vaginoscopy see Transvaginal laparoscopy and endosurgery; Urethrocystoscopy/vaginoscopy

Vascular interventions congenital portosystemic shunts (PSSs) 274-5 interventional oncology 273-4

intrahepatic shunt embolization 274-5

percutaneous transvenous embolization (PTE) 275

Vascular ring anomalies 52

Vasectomy 202-3

Veress needle 24 technique 114, 186-7

Vestibule, pathological conditions 171-2

Vestibulovaginal stenosis 172

VETPUMP ${ }^{\circledR} 2$ system 24,27

Video-endoscopes

comparison with fibrescopes 14

controls 39

imaging systems $24-5,45$

structure 12

Video extras

flexible endoscopy, basic technique

escape of air bubbles during a leakage test of an endoscope 34

single- and two-handed manipulation of steering wheels 38

steering by tip deflection and torqueing 38

laparoscopy

passing instruments around the caudal edge of the falciform fat 201

oesophagoscopy

the endoscope in a j-flexed position looking at the lower oesophageal sphincter (abnormally dilated) in a patient presenting with chronic vomiting. A case of sliding hiatal hernia 51

otoendscopy

flushing technique: appearance of the intact tympanic membrane after the flushing procedure 163

flushing technique: flushing and suctioning cycles in the external ear canal of a dog with otitis externa 162 foreign bodies: removal of plant awns using grasping forceps passed through the working channel of the video-otoscope 158

myringotomy procedure: the incision into the caudoventral quadrant of the pars tensa is made with a 3.5 Fr tomcat catheter 164

removal of an inflammatory polyp from the ear canal of a cat 159

respiratory tract endoscopy

bilateral laryngeal paralysis in a cat 102

endoscopic removal of a grass stalk foreign body from the airway of a dog 110

epiglottic retroversion - intermittent spontaneous retroflexion of the epiglottis during inspiration causing obstruction of the rima glottides 101

grade III collapsing trachea in a Chihuahua $103 \bullet$ laryngeal carcinoma in a cat 102

thoracoscopy

demonstration of the correct bronchoscopically guided placement of an endobronchial blocker 221

optical entry using a trocarless cannula (ENDOTIP Karl Storz Endoscopy) 222

resection of a cranial mediastinal mass and removal from the thorax in a specimen retrieval bag 220

resection using an endoscopic stapler of a consolidated lung lobe in a dog secondary to pneumonia associated with chronic grass awn migration 228

thoracic duct ligation with haemoclips 230

transanal (transcolonic) laparoscopy

transanal laparoscopy in a dog 311

transgastric laparoscopy

closure of the transgastric laparocentesis site using an over-the-top technique 309

dog 1 year after transgastric laparoscopy with closure of the laparocentesis site using endoscopic clips 309

transgastric approach to the abdominal cavity in a dog using a hybrid NOTES procedure 311

transgastric cholecystectomy in a dog 309 transgastric gastropexy in a dog (case 1) 310 transgastric gastropexy in a dog (case 2) 310 transgastric ovariectomy in a dog 309

transoesophageal approach to the thorax peroral thoracoscopy in a cat 311

transvaginal laparoscopy and endosurgery

transvaginal approach to the abdomen using a flexible endoscope 306

transvaginal ovariectomy in a cat using a diode laser 308

transvaginal ovariectomy in a dog 307

transvaginal ovariectomy in a dog using an optical trocar 307

transvaginal ovariohysterectomy in a dog 308 transvaginal ovariohysterectomy in a dog using a hybrid NOTES procedure 311

upper gastrointestinal (Gl) tract endoscopy

placement of a percutaneous endoscopic gastrostomy (PEG) tube in a cat 82

upper $\mathrm{Gl}$ endoscopy 68

upper $\mathrm{Gl}$ endoscopy of a dog with a gastric carcinoma 75

video sequence recorded from an Alicam ${ }^{\circledR}$ video endoscopy capsule, showing a bleeding gastric polyp 71 
urethrocystoscopy/vaginoscopy

normal urethrocystoscopy of the neutered bitch $176 \bullet$

normal urethrocystoscopy of the neutered male dog $177 \bullet$

Video-otoscopy see Otoendoscopy

Wireless endoscopic CCD camera systems $9,70-1$

Xenon light sources 23, 25, 61, 133, 169, 184 